\title{
Limitations and Recommendations for the Measurement of Residual Stresses in Welded Joints
}

\author{
Thomas Noël Nitschke-Pagel \\ Langer Kamp 8, 38106 Braunschweig, Germany \\ t.pagel@tu-braunschweig.de
}

\section{Keywords: Residual Stresses, Welding Joints, X-Ray Diffraction}

\begin{abstract}
Residual stresses in welded joints are often of extended interest in order to evaluate unexpected failures or distortions. Since the possibilities to calculate residual stresses in welds are still stronglyl limited the measurement techniques are still of great importance. Several measurement techniques with particular possibilities and limitations are available today where especially the different diffraction methods are used mostly. Material. weld type and the size of the components are important for the quality of the results obtained with different methods as well as the environment where the measurements have to be carried out. The paper shall give an overview of the results of a round robin test on the application of XRD on butt welded joints which has been carried out in cooperation of different experienced laboratories. The results show the high reliability of XRD-measurements in welds, if the measurements are performed under well defined boundary conditions. The experiences can be used as a recommendation about useful measurement conditions the expectable quality of the results.
\end{abstract}

\section{Introduction}

Residual stresses are mentioned very often if performance problems of welded construction are discussed [2]. Brittle fracture, low fatigue resistance, corrosion or unexpected distortion are related frequently to high tensile residual stresses. However knowledge about the magnitude of residual stresses is rarely precise but mostly assumptive or based on generalizing catalogues [1]. Many efforts are made since more than 30 years to calculate the welding residual stresses but in fact this possibility has not yet been established in practice because calculations are mostly limited to very small and simple weld geometries. Multipass welds or welded components with a high number of single weld seams cannot yet be handled with the numerical tools. Consequently in practice many simplifications are usual followed by a mostly poor reliability. A consideration of residual stresses in design rules reliable quantitative knowledge about the residual stresses in critical zones of a component are required and they should be determined non-destructive[1,2]. In practice XRD is applicable in several variations whereas uncertainty exists often when the customers are not experienced about the recommended conditions and the expectable accuracy of the results.

\section{Background of the RR-test on welds}

Several techniques are applicable to determine residual stresses in welds [3,4]. Mechanical techniques like the hole drilling use a locally restricted access to the material in order to determine the initial residual stresses while destructive sectioning techniques like the contour method allow the determination of the initial residual stresses in the entire cross section with preference to the longitudinal residual stress component. Non-destructive techniques like the micromagnetic method are available but the signals cannot be directly correlated to a strain or stress condition. The signals generally a calibration procedure which may complicate the measurements. The experiences of residual stress evidently show that the most reliable results 
can be expected by means of diffraction [5,6,7]. In addition to X-ray diffraction (XRD) high energy methods with particular advances and limitations are well established nowadays [8]. Limitations for the practical use are the restricted access to the facilities which makes them more useful in fundamental research. XRD is a widely used method with a lot of experiences in different materials and their conditions. Since a lot a different portable devices are available the method is preferable also in welding practice because it can be used under laboratory as well as under rough environmental conditions.

\section{Special problems of XRD-application in welded joints}

Residual stress determination in welded joints is usually complicated by different sources resulting from the microstructure in combination with a more or less complicate geometry. Undercuts or sharp notch geometry at the weld toe and rough surface in the weld seam as well as bulging weld material lead to difficult adjustment conditions. In combination with coarse grain this ends in higher scattering range or incorrect results. Slag rests or condensed metal dust may cover the surface and act like beam absorber. Frequently the exposure time must be extended strongly but anyway the scatter range of the results will arise. Sometimes this problem can be solved by reducing the local resolution but frequently in such zones XRDmeasurements are impossible like in the weld zone of Al- or Ni-based alloys or high alloyed steels.

A simple problem is generated by the shape of the weld. As Fig 1 illustrates the weld material may build a barrier for the incident or the reflecting beam which must end in an error. In butt welds the expected problems will be detachable as long as the height of the bulged weld material is not too high. In T-joints or welds with fillet welded longitudinal
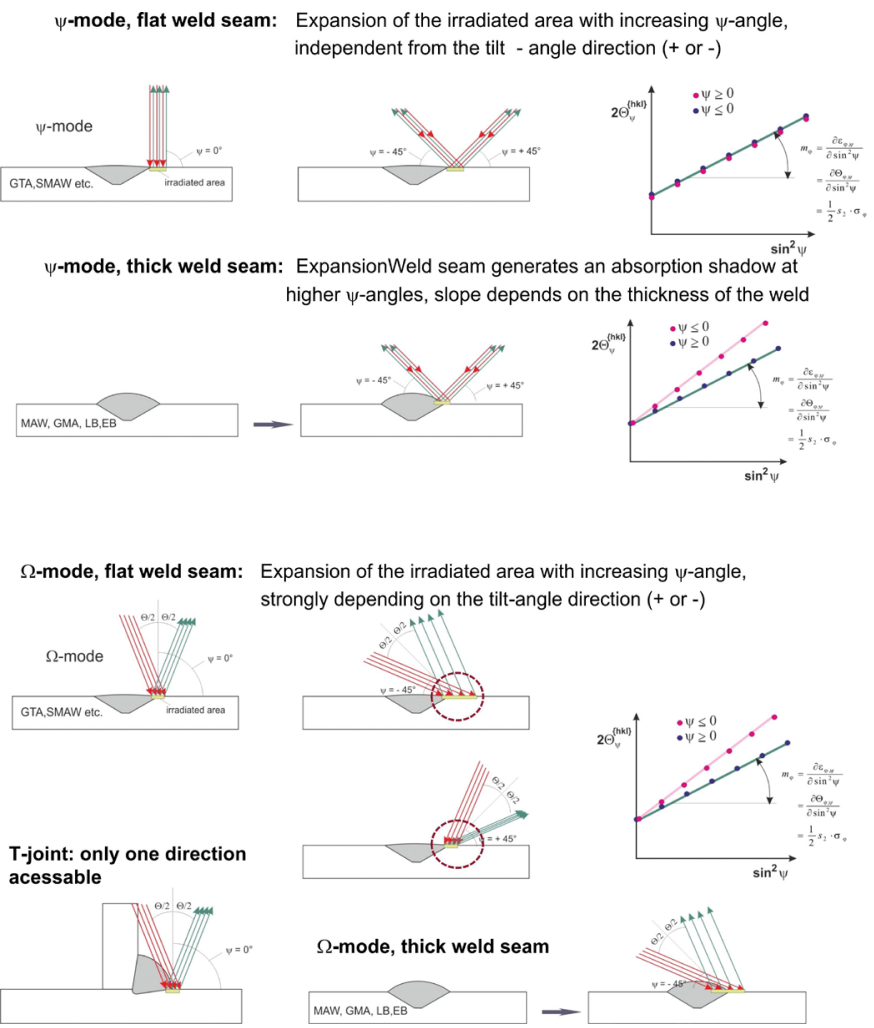

Fig 1. Relationship between weld shape and beam geometry with regard to the accessibility of the $\sin ^{2} \psi$-method. stiffeners the weld toe and the connected stiffener build a barrier which limites the measurement on one $\psi$-direction.

This is a serious problem because the peak determination in both $\psi$-tilt directions is a criterion for fulfilling the preconditions of the $\sin ^{2} \psi$-method. Since in practice in welds with geometrical restrictions the adjustment of a precise $\psi$-startposition 0 at the measured point is not always possible as good as required. Here the movement in positive and negative $\psi$-direction enables the determination of a reliable average value. Limitation on one direction on the other hand will lead to an error depending on the discrepancy at the zero position and fin ally to a more or less greater uncertainty of the determined residual stress value. However it must be accepted that in welds with stiffeners movement in both directions is impossible. Then special attention must be payed for an adjustment of the sample as precise as possible. 


\section{Round-Robin-Test on residual stress determination using XRD}

Purpose of the Round-Robin-Test

An approach to establish residual stresses in welding design codes requires first an answqer on two basic questions. The first question is the possible and the required local resolution, the second is the accuracy.

The applicable resolution is mostly defined by the used devices and by the material condition. The technical range of applicable collimator diameters starts at approximately $0.3 \mathrm{~mm}$ and ends at some mm. For special applications also much smaller diameters can be used but this is usually not recommended for welded joints. The experiences of the practical application of XRD reveal that normally collimator sizes lower than $1 \mathrm{~mm}$ lead to higher scattering and large extensions of the required exposure times. Diameters higher than $3 \mathrm{~mm}$ are not useful because lateral surface residual stress gradients in the surrounding of a weld seam may be smoothened strongly. In practice diameters e.g. local resolutions of $1 \ldots 2 \mathrm{~mm}$ represent a good compromise of an acceptable resolution and a limited time effort for exposure. Of course this agreement is useful for standard arc welds while in very narrow beam welds this limitation may be not acceptable (Fig.2).

Standard rules for XRD-residual stress
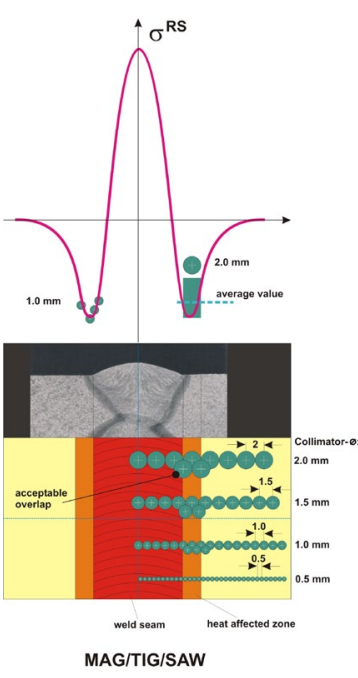

$$
\text { Example: Steel, plate thickness } 10 \mathrm{~mm}
$$

Fig 2. Influence of the collimator size on the local resolution of residual stresses and smoothina effect.

measurements are defined in [9], where recommendations for the measurement, the calculation procedure and useful material parameters as well as recommendations for the required calibration proceduresare are summarized. The careful consideration of the standard rules should guarantee that measured results are independent from the lab or the operator. This basic request was the initial idea of the presented RR-test where the quality of typical residual stress determination in standard welds performed by different experienced labs should be demonstrated.

\section{Examined objects}

The experiments were carried out using flat plates $\left(200 \times 150 \times 6 \mathrm{~mm}^{3}\right)$ of a normalized fine grained normalized structural steel (german grade S460N) with a yield strength of $460 \mathrm{~N} / \mathrm{mm}^{2}$. The plates were planely ground and stress relief annealed before welding at $600^{\circ} \mathrm{C} / 10 \mathrm{~min}$. A TIG-welded dummy seam was generated with a heat input of $10.92 \mathrm{~kJ} / \mathrm{cm}$ As Fig 3 shows the TIGprocess generates a very flat weld seam while the MAG-process leads to a strongly bulged weld seam with difficult geometrical measurement conditions. Due to the relatively low heat input the

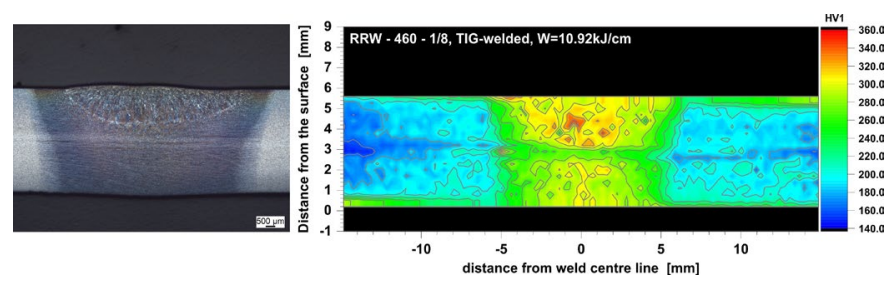

Fig 3. Weld geometry, structure and hardness distribution. (HAZ) are rather similar. 


\section{Results of the Residual stress measurements}

Fig.4 (top) shows the representative distributions of the longitudinal and transverse residual stresses across the weld seam of a TIG-welded sample determined with a commonly used collimator- $\varnothing$ of $1.0 \mathrm{~mm}$. Here the typical symmetrical residual stress profiles are observed with some minor discrepancies in the weld material and the typical maximum and minimum peaks in the transition zone between the weld and the parent material. The average measurement error is below $10 \mathrm{MPa}$ except in the weld seam where an error level of 50 $\mathrm{MPa}$ is reached. The error of the transverse residual stresses is necessarily higher. The reason is that due to the shift of the $\psi$-angle the geometry of the irradiated area changes with $\psi$ and therefore the
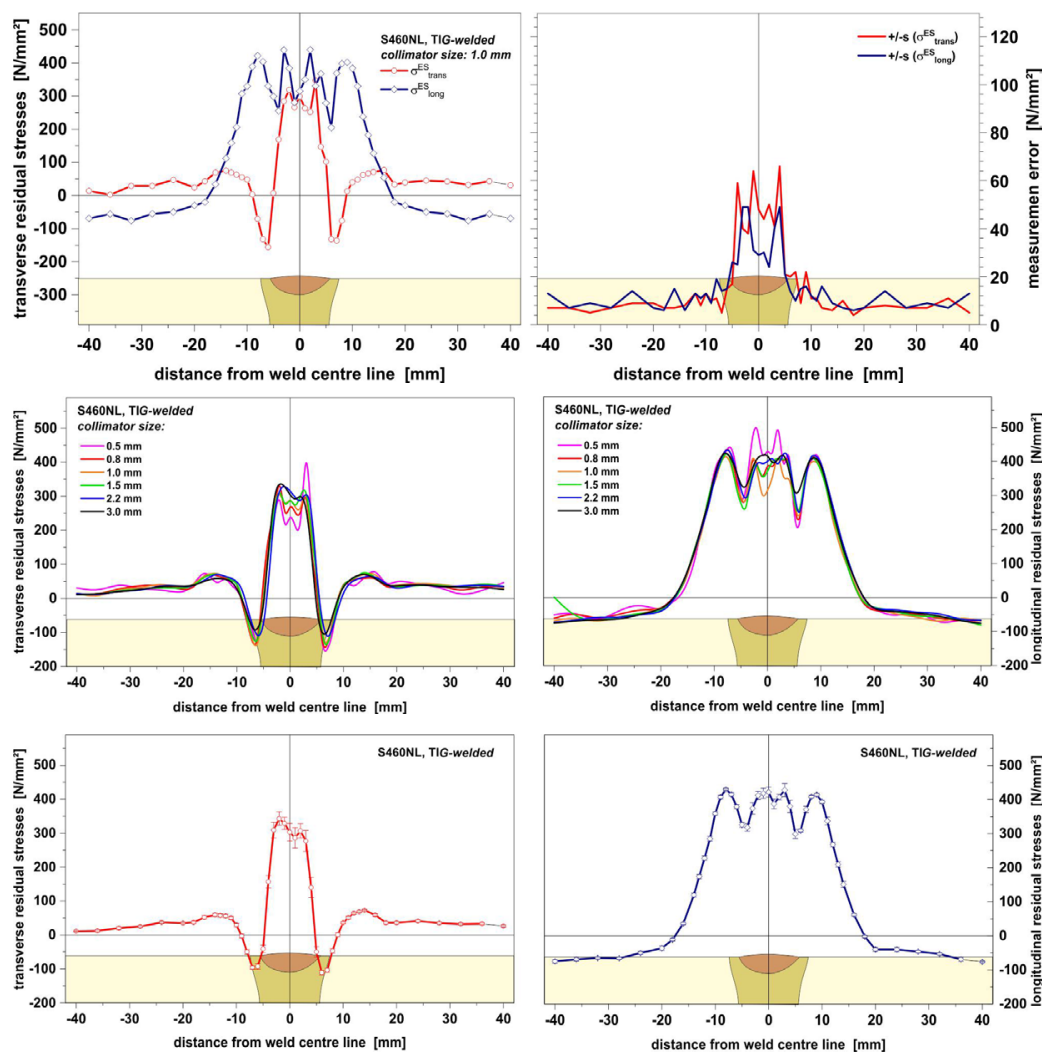

Fig 4. Comparison of the residual stress profiles measured with different local resolutions (collimator- $\varnothing$ ), average distributions and error-range. measured value is more influenced by the stress gradient. In longitudinal direction the interval of the stress gradient is constant which leads to a lower error.

Influence of the local resolution on the quality of the measurement results

The influence of the local resolution on the measured residual stress profiles is represented in Fig.4 (middle) where the obtained profiles using collimators with diameters from 0.5 to $3.0 \mathrm{~mm}$ are compared. The measured results are obviously independent from the collimator size except the smallest diameters of 0.5 and $0.8 \mathrm{~mm}$. Here a greater discrepancy in the weld material is present. However the deflection of the profiles with particular peaks and tales in the transition zone between the weld and the parent material is marginal in longitudinal and in transverse direction. The average values with the related error bars are shown in Fig.4 (bottom). The divergence in the weld seam is below $\pm 30 \mathrm{~N} / \mathrm{mm}^{2}$. This is of great importance because it demonstrates that the residual stresses in arc welds can be measured with a good accuracy without the requirement of very high local resolution. Since the local resolution is very important for the exposure time and that is to say for the time effort for a single measurement this results offer the possibility to determine residual stresses in welds in an acceptable time span. 
Results of the RR-tests

In Fig 5 (top) the residual stress distributions measured in TIG-welded plates by the participating labs results using uniform collimator sizes of 1.0 $\mathrm{mm} \varnothing$ are shown. As the resulting average profiles with the resulting scatterband reveal (Fig.5, bottom) the scatter range in the base material and in the heat affected zone is between $\pm 25 \mathrm{~N} / \mathrm{mm}^{2}$ and $\pm 40 \mathrm{~N} / \mathrm{mm}^{2}$. In the weld seam increasing deviations with an amount of $\pm 100 \mathrm{~N} / \mathrm{mm}^{2}$ are present. Neverthe-less the agreement of the obtained residual stress profiles can be interpreted as
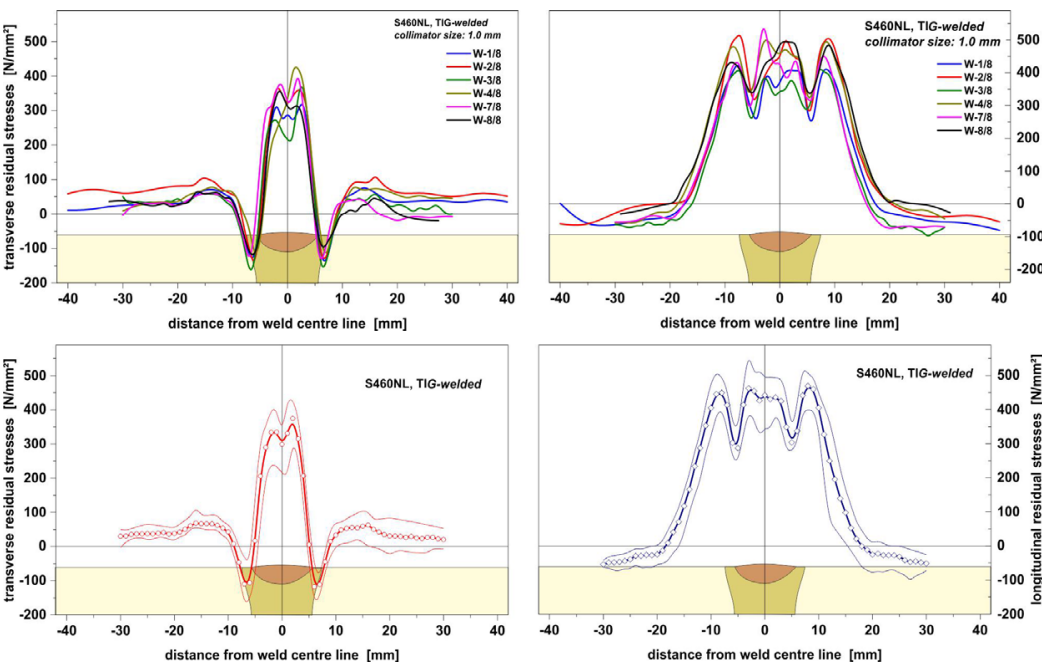

Fig 5. Comparison of the residual stress profiles obtained by the participating laboratories, average profiles and related error ranae.

excellent. Experiences with larger numbers of specimen of the same series, have shown that the variation of the residual stresses in welds may vary between $\pm 20 \ldots \pm 80 \mathrm{~N} / \mathrm{mm}^{2}$ even if the specimen have been produced under comparative laboratory conditions. Considering this unpreventable variation the agreement of the obtained results is quite good. Larger deviations of the different measurements are connected with the integral width of the measured diffraction lines (Fig.6, left hand side). This is not really surprising because these values are much more influenced by the particular devices and assessment procedures (detector
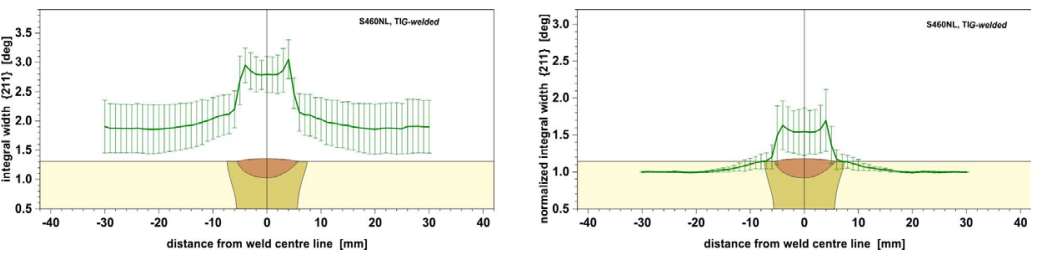

Fig 6 Comparison of the measured and normalized profiles of the integral width of the diffraction lines samples. type, beam geometry,peak smoothing, etc) If the determined integral widths are normalized using the corresponding values of the base material this error is reduced significantly. Nevertheless in the weld material a greater discrepancy must be accepted.

\section{Summary and conclusions}

The determination of residual stresses by means of X-ray diffraction is the most suitable technique if confidential results are expected without any detraction of the investigated components even under rough or difficult environmental conditions. However the accuracy and the reliability of the obtained results depends strongly on the adherence of some standard [10] rules considering the special requirements of welds:

- $\quad$ a sufficient number of $\psi$-angles is required to eliminate the disturbing effects of coarse grain and unsteady surface in the irradiated area in the surrounding of the weld seam.

- measurement in both $\psi$-direction is generally recommended. The consideration of both directions enables to compensate smaller adjustment errors.

- in welds with a bulged weld seam or shading stiffeners, where measurements cannot be performed in both $\psi$-directions a higher precision for the adjustment is strictly required. 
- in a technical useful range the chosen resolutions do not affect strongly the accuracy of the measured residual stresses. In the weld seam the detrimental effect of the grain structure compensates the benefit of a higher resolution by scattering. Useful collimator sizes of $1 \ldots 2$ $\mathrm{mm}$ combine a sufficient local resolution with a lower error and an acceptable exposure time.

Repeatable measurement results within a range of $\pm 50 \mathrm{~N} / \mathrm{mm}^{2}$ or better can be expected independent from the operator or the used equipment. This perception is applicable for conventional arc welds with technically usual weld seam geometries. A proval of a general applicability for welds including small beam welds and comparable should be given separately.

\section{Acknowledgement}

The RR-test was carried out with help of the collaboration of René Fenzel (SLV Halle, Germany), Jens Gibmeier (KIT, Germany), Paul Lefèvre (SONATS, France), Fabien Lefebvre (CETIM, France) and Wolfgang Zinn (University of Kassel, Germany). The author wants to express his thank for the participation and helpful contributions.

\section{References}

[1] Barthelmey, J.Y.; Janosch,J.J.: Structural Integrity Assessment Procedure for Euopean Industry - SINTAP, Task 4 Compendium on Residual Stress Profiles. Brite-Euram-Sintap, BRPR-CT95-0024, 18.05.1999

[2] Bate, S.K.; Green,D.; Buttle,D.: A review of residual stress distributions in welded joints for the defect assessment of offshore structures. Offshore technology report, OTH 482, HSE Books 1997

[3] Lu, J.: Handbook of Measurement of Residual Stress, Society for Experimental Mechanics Inc., American Institute of Physics, 1996

[4] Bahadur,A.; Kumar,B.R.; Kumar, A.S.; Sarkar,G.G.; Rao, S.: Development and comparison of residual stress measurement on welds by various methods. Materials Science and Technology, Volume 20, 2004 - Issue 2, pp261-269

[5] I. C. Noyan, J. B. Cohen. Residual stress - measurement by diffraction and interpretation. Springer Series on Materials Research and Engineering, edited by B. Ilschner and N. J. Grant. Springer-Verlag, 1987.

[7] Eigenmann, B.; Macherauch, E.: Röntgenographische Untersuchung von Spannungszuständen in Werkstoffen. Materialwissenschaft und Werkstofftechnik Band 26 (1995). Teil I.; Heft 3; S. 148-160.; Teil II. Heft 4, S. 199-216; Teil III. Heft 9, S. 426-437

[8] Spiess, L.; Teichert, G.; Schwarzer, R.; Behnken, H.; Genzel, C.: Moderne Röntgenbeugung - Röntgendiffraktometrie für Materialwissenschaftler, Physiker und Chemiker: Springer Verlag 2009. https://doi.org/10.1007/978-3-8349-9434-9

[8] M.E. Fitzpatrick; A.T. Fry; P. Holdway; F.A. Kandil; J. Shackleton; L. Suominen: Determination of Residual Stresses by X-ray Diffraction - a national measurement good practice guide, Measurement Good Practice Guide No. 52, National Physical Laboratory Teddington, Middlesex, United Kingdom, 2005

[9] DIN EN 15303 Non-destructive testing - Test method for residual stress analysis by X-ray diffraction; German version EN 15305:2008 